Revista Mexicana de Economía y Finanzas Nueva Época

Volumen 14 Primer Número Especial Aniversario, Agosto 2019, pp. 527-540

DOI: https://doi.org/10.21919/remef.v14i0.421

\title{
Spillovers entre el S\&Poor500 y los principales EMBIG latinoamericanos
}

\author{
Francisco López-Herrera ${ }^{1}$ \\ Universidad Nacional Autónoma de México, México \\ Domingo Rodríguez Benavides \\ Universidad Autónoma Metropolitana, México \\ César Gurrola Ríos
}

Universidad Juárez del Estado de Durango, México

(Recepción: 26/septiembre/2018, aceptado: 22/marzo/2019)

\section{Resumen}

En este trabajo se realiza un análisis de los efectos de derrame (spillover) entre los rendimientos accionarios de Estados Unidos y los cambios en los índices EMBI Global de Argentina, Brasil, Colombia, México y Perú. Se estima un índice del spillover total que muestra alzas importantes a principios de siglo y durante la crisis financiera mundial. Al descomponer dicho índice en sus componentes direccionales se observa que las principales fuentes de spillover entre los mercados analizados son el mercado accionario de Estados Unidos y el índice de los bonos brasileños. Una limitación importante es que quedan fuera del estudio los EMBIG de otros países latinoamericanos y la principal implicación de los resultados es que muestra cómo se relaciona el comportamiento de esos instrumentos, lo que puede ser útil para la toma de decisiones de portafolio. También es de destacarse que los principales receptores de efectos derrame son los índices de los bonos de Perú y México, en tanto que el índice de los bonos argentinos son los menos afectados por los choques externos.

Clasificación JEL: F21, F36, F65, G10, G11, G15

Palabras clave: spillover, derrames, EMBI, S\&P500, bonos soberanos

\section{Spillovers between the S\&Poor500 and the top Latin American EMBIG}

\section{Abstract}

This work performs an analysis of spillover effects between U.S. stock returns and changes in EMBI Global indices in Argentina, Brazil, Colombia, Mexico and Peru. A total spillover index is estimated to show significant increases at the turn of the century and during the global financial crisis. Breaking down the index into its directional components shows that the main sources of spillover among the markets analyzed are the U.S. stock market and the Brazilian bond index. An important limitation is that the EMBIGs of other Latin American countries are left out of the study and the main implication of the results is that it shows how the behavior of these instruments relates, which can be useful for portfolio decision-making. It is also noteworthy that the main recipients of spill effects are the indices of the bonds of Peru and Mexico, while the Argentine bond index is the least affected by external shocks.

JEL Classification: F21, F36, F65, G10, G11, G15

Keywords: spillover, Spills, EMBI, SEP500, Sovereign Bonds

\footnotetext{
${ }^{1}$ Cubículo 44, División de Investigación, FCA-UNAM, Circuito Exterior S/N, Cd. Universitaria, Coyoacán, 04510, Cdmx. Teléfono 5622 8494, francisco opez $_{h}$ errera@yahoo.com.mx
} 


\section{Introducción}

La familia de los índices de bonos de los mercados emergentes (EMBI) fueron creados por J. P. Morgan, proporcionando en la actualidad información diariamente de una canasta de instrumentos de deuda emitidos por gobiernos, bancos y empresas de países llamados emergentes y con un historial que empieza para algunos instrumentos a partir de diciembre 31 de 1990. Su objetivo es proporcionar a los inversionistas elementos de referencia para evaluar el desempeño y analizar los rendimientos y riesgo de bonos soberanos denominados en dólares de Estados Unidos y con un valor en circulación calculado en al menos 500 millones de dólares, excluyendo emisiones que no se encuentran a la disposición del público inversionista o que no cuentan con niveles de liquidez que esa empresa considera convenientes. Los EMBI miden el diferencial (spread) en puntos bases de la tasa de interés que pagan los emisores de bonos y los Treasury Bills de Estados Unidos, considerados teóricamente como "libres de riesgo". El índice EMBI Global incluye a países altamente calificados con base en los niveles del ingreso per cápita definidos por el Banco Mundial y el historial del país en cuanto a la reestructuración de su deuda.

En este trabajo se analizan las interrelaciones entre los índices EMBI Global de Argentina, Brasil, Colombia, México, Perú y el ampliamente conocido índice accionario de Estados Unidos S\&P500. El objetivo es averiguar cómo se influyen los diferentes mercados analizados y cómo los choques del mercado accionario de Estados Unidos se derraman hacia esos índices de bonos. La siguiente sección se encarga de llevar a cabo una revisión de la literatura seguida por una exposición resumida de la metodología empleada posteriormente para la cuantificación de los efectos de derrame (spillover) y el análisis de los spillovers causados por los choques del mercado accionario de Estados Unidos. Finalmente, se presentan las conclusiones.

\section{Revisión de literatura}

Tal vez haya poco que agregar a la reiterada afirmación de que el mundo actual refleja los efectos de una sociedad globalizada y que, tales efectos junto al nivel de dicha globalización son cada vez más profundos y por ende, más fácilmente observables; sin embargo, lo que si adquiere notoria importancia es el análisis y comprensión de las consecuencias que aquella ocasiona sobre las decisiones de inversión y financiamiento de los distintos agentes económicos. En la literatura financiera es posible identificar, entre los aspectos que han cobrado especial importancia en la agenda de investigación internacional, por el impacto directo que la tendencia global mencionada líneas atrás tiene sobre el crecimiento económico, la desregulación de los mercados financieros y la libre movilidad de capitales (Krueger, 1980; Iglesias, 1992; Te Velde, 2003; Oliva y Suarez, 2007; Kristjanpoller y Salazar, 2016).

Desde la última parte del siglo pasado los gobiernos de distintas economías, principalmente emergentes, han venido adoptando una serie de reformas estructurales con la intención de lograr y mantener estabilidad macroeconómica mediante el mejoramiento en sectores clave, entre ellos el laboral y el financiero. Entre las estrategias adoptadas para tal efecto destaca el fortalecimiento de los vínculos comerciales hacia el exterior, reducción de aspectos regulatorios así como importantes procesos de privatización (Cimoli y Correa, 2005; Te Velde, 2003; Iglesias, 1992). Al margen de los resultados oficiales reportados como consecuencia de la implementación de dichas reformas vale la pena señalar que éstos han sido cuestionados por su impacto diferenciado señalando por ejemplo, que dichas reformas liberales pudieran tener influencia negativa sobre indicadores de empleo, salarios, tipo de cambio, derechos de los trabajadores y que dichos efectos negativos impactan en mayor medida en los sectores de menor ingreso (Onaran, 2009; Oliva y Suárez, 2007). Otros factores a considerar para evaluar holísticamente el éxito de las reformas estructurales debieran incluir, además de ciclos económicos que pueden incidir en la presencia 
Revista Mexicana de Economía y Finanzas Nueva Época, Vol. 14 Primer Número Especial Aniversario, pp. 527-540

de rompimientos estructurales, asimetrías entre las condiciones de mercado de distintas regiones, el impacto de innovaciones tecnológicas en productividad, competitividad y comercio; factores medioambientales (Bielschowsky, 2009; Oliva y Suarez, 2007) y por supuesto, los mecanismos y la velocidad de transmisión de efectos económicos y financieros (Santillán, Gurrola, Jiménez y Venegas, 2018). Vale la pena destacar, en este momento, que el contagio no sólo se explica por las crisis económicas nacionales sino también por shocks o perturbaciones de otra índole, como el incremento de los precios internacionales del petróleo del 2000 y los efectos del desplome del mercado bursátil NASDAQ en 2001 (Frenkel, 2003), y más recientemente, el efecto nocivo de la crisis gestada en el mercado de hipotecas de alto riesgo en los EUA, estos dos últimos son ejemplos de la probada influencia que el mercado de capitales de la economía estadounidense tiene sobre los fundamentales macroeconómicos de otros menos desarrollados.

La apertura comercial ha sido señalada como un factor detonante de los procesos de crecimiento, con especial énfasis en economías emergentes (Krueger, 1980). No obstante lo anterior, la intensificación de los vínculos comerciales también puede estar asociada con el incremento de los niveles de interdependencia entre el precio de activos financieros que cotizan simultáneamente de distintos mercados, generando e intensificando canales de contagio o transmisión de efectos económicos entre ellos (Calvo y Reinhart, 1996; Hernández y Valdés, 2001). Por otro lado, diversos estudios han documentado en la región de América Latina que el fortalecimiento del comercio exterior también contribuye a generar espacios de integración financiera que, no obstante la reducción en los costos de transacción y en el costo de capital, pueden entorpecer las decisiones de inversión del capital internacional en su intento por reducir los niveles de exposición al riesgo sistemático doméstico, vía la diversificación de portafolio (Romero et al, 2013; Sandoval y Soto, 2016; Mellado y Escobari, 2015).

Ente los temas cuya importancia se ha venido incrementando en los últimos años, con especial énfasis en la región de América Latina, destaca el estudio de los procesos en que las perturbaciones observadas en un mercado se transmiten a otras economías, lo anterior en función del impacto que tales procesos de contagio tienen sobre los flujos de inversión internacional (Rigobon, 2002; Frenkel, 2003; Mollah y Hartman, 2012; Marcet, 2017). La creciente importancia del contagio financiero radica, entre otras causas, en la aparente disminución de las posibilidades de consolidar portafolios diversificados en la medida en que los vínculos de integración financiera ente economías y mercados se van fortaleciendo. Si bien es cierto que el término "contagio" se ha popularizado en la literatura financiera habría que señalar que su origen y uso, en el sentido descrito en las líneas anteriores, es relativamente reciente pues está asociado a las crisis financieras de los últimos años del siglo XX. Entre algunos de los estudios pioneros que abordan la discusión sobre la propagación de efectos entre mercados financieros destacan Calvo y Reinhart (1996), Rigobón (2002), Forbes y Rigobon (2002).

Para inicios de los noventa del siglo pasado, el tema de la forma en que economías emergentes, particularmente de América Latina, abiertas al mercado internacional, eran afectadas por el desempeño de otras economías así como a través de los mercados financieros, era un fenómeno poco estudiado y que la relevancia del término contagio comienza a reposicionarse a partir de la crisis de México en 1994 y la forma en que sus efectos se percibieron en otras economías emergentes de la región y en Asia (Calvo y Reinhart (1996). ${ }^{2}$ El estudio, al analizar los canales de transmisión y determinantes de la intensidad del contagio propone, entre las posibles explicaciones aspectos como el nivel de integración de los mercados de capital, derrames de volatilidad, acuerdos comerciales, prácticas institucionales, sesgo por mercados desarrollados, factores tecnológicos e inesta-

\footnotetext{
${ }^{2}$ Para una revisión detallada de crisis financieras de finales del siglo XX revisar Calvo y Reinhardt (1996) y Rigobon (2002), para la crisis mundial de la década anterior Mendoza, López y Watkins (2011) y Santillán (2015).
} 
bilidad política. Los autores hacen un llamado importante sobre el tema de los spillovers, particularmente para economías emergentes que tienen la intención de mejorar sus niveles de apertura comercial, ya que las hace potencialmente vulnerables no sólo a la evolución de factores económicos externos como los ajustes a los términos del comercio, tasas de interés internacionales, sino también a acontecimientos económicos y políticos observados en los países vecinos.

Rigobon (2002) aborda la transmisión de efectos entre mercados financieros mediante el análisis de colapsos financieros, entre ellos el desplome del mercado bursátil de EUA en 1987, la devaluación de peso mexicano en 1994, la caída de la bolsa de Hong Kong en 1997, y confirma que tales eventos afectaron, de alguna manera, la dinámica de los mercados financieros internacionales. Sin embargo el autor hace una llamado a interpretar dichos resultados con cautela, llegando incluso a proponer en lugar de "contagio" el término de "interdependencia", al sostener que el contagio es más que la sola presencia de una alta correlación entre mercados, aun cuando ésta se vea incrementado después de perturbaciones inesperadas en alguno de ellos.

Diversas metodologías de análisis lineal han sido utilizadas con la intención de modelar el contagio, entre las más utilizadas destacan el coeficiente de correlación, el análisis de componentes principales y el procedimiento de mínimos cuadrados. No obstante la popularidad de tales técnicas es preciso que señalar entre sus principales debilidades, su alta sensibilidad a la volatilidad observada, lo cual puede ocasionar que en medio de las turbulencias financieras, donde se esperaría presenciar un importante incremento, el parámetro estimado sobreestime el nivel de asociación entre los mercados bajo estudio; lo anterior sin mencionar las limitaciones ocasionadas por las propiedades estadísticas de las series financieras -heterocedasticidad, autocorrelación y ausencia de normalidad (Forbes y Rigobon, 2002).

Un análisis completo sobre contagio financiero debiera incluir, al menos, las siguientes consideraciones: a) el riesgo de no capturar adecuadamente la heterocedasticidad de la información, lo que ocasiona sesgos importantes que pueden sobrevalorar las interacciones normales entre los mercados, b) la existencia de efectos posteriores a los shocks inesperados que son perfectamente normales y que, en ocasiones, se confunden con contagio, y c) si bien es cierto que la mayor parte de la transmisión de los shocks puede ser atribuible al comercio, es importante reconocer que una buena parte puede explicarse ante una realidad en que los activos analizados se cotizan simultáneamente en los mercados bursátiles entre los que se asume el contagio (Rigobon, 2002).

Así las cosas, también es posible identificar estudios que abordan el tema del contagio financiero haciendo uso de técnicas de análisis más robustas, por ejemplo Uribe (2011) a partir de un modelo de valoración internacional de activos por factores e incluyendo las fortalezas estadísticas de las cópulas y la teoría del valor extremo, propone una metodología para medir el contagio a través de coeficientes de dependencia asintótica; más recientemente Santillán, Gurrola, Jiménez y Venegas (2018) también utilizan la metodología de cópulas bivariadas para analizar las relaciones de interdependencia entre las colas de las distribuciones de los índices bursátiles, en los principales mercados de América Latina.

Otro tema que ha venido cobrando especial importancia en la literatura financiera es el premio al capital extranjero que un país debe ofrecer a fin de compensar apropiadamente el riesgo del mercado doméstico, tal importancia podría explicarse, al menos en parte, ante el incremento en el acceso de los mercados emergentes al financiamiento internacional y de la presencia de crisis económicas de los últimos 30 años. Adicionalmente a lo anterior, dicho indicador presenta afectaciones potenciales sobre las decisiones de política monetaria de los países al establecer restricciones debido a la relación entre el riesgo país y el equilibrio del saldo de la balanza con el sector externo (López, Venegas y Gurrola, 2013). También, habría que considerar el hecho de que las inversiones provenien- 
Revista Mexicana de Economía y Finanzas Nueva Época, Vol. 14 Primer Número Especial Aniversario, pp. 527-540

DOI: https://doi.org/10.21919/remef.v14i0.421

tes de mercados desarrollados hacia países emergentes han generado pérdidas millonarias como consecuencia de distintos aspectos asociados al riesgo país, entre ellos: violaciones regulatorias, corrupción, fraudes o daños a la reputación (Horn et al, 2017).

El riesgo país se asocia con la probabilidad de que un país no cumpla sus obligaciones financieras con acreedores internacionales así pues, los agentes económicos que acuden a los mercados financieros internacionales a solicitar fondos se ven sujetos a una sobretasa en función a tal probabilidad de incumplimiento. La percepción del riesgo país tiene efectos importantes en el costo de financiamiento para las empresas del mercado doméstico al repercutir en el flujo de capitales, ya sea en activos reales o financieros, en ambos casos un inversionista (individual o institucional) debería de contar con estimaciones precisas de los niveles de riesgo país, especialmente de aquellas economías sujetas a mayores niveles de volatilidad que sus contrapartes desarrolladas (Lessard, 1996, Godfrey y Espinosa, 1996).

La sobretasa mencionada en las líneas anteriores representa la diferencia del rendimiento ofrecido por los bonos domésticos y la tasa que pagan los bonos del Departamento del Tesoro de Estados Unidos. Un referente comúnmente aceptado para representar el riesgo país es el Emerging Market Bond Index, EMBI, índice construido por J.P. Morgan para para describir la evolución del riesgo de la deuda soberana emitida por mercados emergentes, razón por la cual se puede considerar también que el EMBI es un referente del costo de pedir prestado, junto a otras condiciones, fondos internacionales que se canalizan al sector privado de los países emergentes (Mántey y Rosas, 2014; López, Venegas y Gurrola, 2013; Caporale, Carcel y Gil-Alana, 2015, Dimic, Kiviaho, Piljak y Äijö, 2016; Özmen y Yaşar, 2016). Vale la pena señalar que J.P. Morgan, en el intento de caracterizar la deuda de los países ofrece dos productos. En primer lugar, el EMBI+, cuyos instrumentos deben tener un mínimo de $\$ 500$ millones en circulación, captura el rendimiento total de los instrumentos de deuda externa negociados incluyendo además del rendimiento de instrumentos de los mercados locales en dólares estadounidenses, préstamos, eurobonos y bonos Brady, denominados en moneda extranjera. El segundo producto con el que J.P. Morgan propone capturar el riesgo país es el EMBI Global, el cual es un índice más amplio pues incrementa la base de datos del EMBI+ al incluir, para su cálculo, activos financieros con un menor nivel de liquidez. Así, el EMBI Global está disponible para un mayor número de países (ampliando la base de 6 a 27), no obstante, presenta una limitante: se empezó a construir a partir de 1998 (Hernández y Valdéz, 2001).

$\mathrm{Al}$ igual en otros campos de la investigación financiera, la mayor parte de los estudios sobre las relaciones entre el riesgo país, capturado por el EMBI, y diversos aspectos de la dinámica económica ha sido aplicado primordialmente en economías desarrolladas, por ejemplo Caporale, Carcel y Gil-Alana (2015); Almansour, Aslam, Bluedorn y Duttagupta (2015); Dimic, Kiviaho, Piljak y Äijö (2016); Özmen y Yaşar (2016), Tillmann (2016), por mencionar sólo algunos. En general, dichos trabajos documentan el efecto significativo del riesgo país sobre fundamentales macroeconómicos y resaltan la importancia del adecuado monitoreo de las primas de riesgo capturadas por el EMBI, así como el reconocimiento de los factores que inciden en su determinación.

No obstante lo anterior, también existen estudios sobre la primas de riesgo país el contexto de los mercados emergentes. Piljak (2013) al estudiar el comportamiento de bonos gubernamentales en Brasil, China, Malasia, México, Perú, Filipinas, Polonia, Rusia, Sudáfrica, Turquía, Argentina, Bulgaria, Colombia y Ecuador, asegura que los factores domésticos, particularmente los asociados a política monetaria e inflación, son más importantes que los externos al momento de dar cuenta de las variaciones conjuntas de los bonos analizados. Mántey y Rosas (2014) sugieren que la estabilidad cambiaria y el comercio internacional impactan en las expectativas de mercado capturadas por el EMBI de las 12 economías emergentes para las que existe información sobre el indicador de riesgo país: Argentina, Brasil, Bulgaria, Colombia, Ecuador, México, Panamá, Perú, Filipinas, Rusia, Turquía y Venezuela. Almansour, Aslam, Bluedorn y Duttagupta (2015) 
REMEF (The Mexican Journal of Economics and Finance)

con una muestra de 16 mercados emergentes, representantes de tres cuartas partes de la producción total de este tipo de economías, mediante un modelo SVAR documentan la vulnerabilidad de las economías estudiadas a shocks provenientes de mercados más desarrollados. Dimic, Kiviaho, Piljak y Äijö (2016) analizan los mercados de Argentina, Brasil, Bulgaria, Colombia, México, Perú, Filipinas, Rusia, Turquía y Venezuela, con la ayuda de la metodología wavelet para concluir que las variaciones en los fundamentales de la economía doméstica afectan las correlaciones acciones-bonos y que tal relación varía significativamente a lo largo del tiempo. Özmen y Yaşar (2016) utilizando una muestra de 23 economías emergentes destaca que los ratings de la deuda junto a las condiciones financieras globales son factores significativos para explicar los EMBIG; asimismo que los spreads no son invariables ante episodios especulativos y de grado de inversión, las transiciones entre ellos y las crisis financieras en otros mercados en desarrollo.

Si bien es cierto que la evidencia de esfuerzos por explorar la importancia de las primas de riesgo del EMBI, particularmente en la región de América Latina es escasa, existen resultados interesantes. López, Venegas y Gurrola (2013) documentan en el mercado mexicano relaciones de largo plazo y spillovers entre variables representantes del riesgo sistemático doméstico y su correspondiente EMBI+. Caporale, Carcel y Gil-Alana (2015) caracterizan el comportamiento estadístico de los EMBI mediante la metodología de integración fraccionaria y documentan, entre los principales resultados persistencia de largo plazo, falta de linealidad y la presencia de rompimientos estructurales. Ortiz, López, Santillán y Fonseca (2016), mediante la metodología de Cópulas bivariadas, reportan un nivel de segmentación importante en la región -Argentina, Brasil, Chile, Colombia, México y Perú-, lo que sugiere la existencia de atractivas oportunidades de diversificación a las inversiones internacionales.

Finalmente, es importante considerar la influencia entre los mercados de capitales y las primas de riesgo país en economías emergentes (Agliardi et al, 2012; Oliveira, Curto y Nunes, 2012; Hassan y Wu, 2015). Para el caso específico de México, Cermeño y Solís (2012) aseguran que el mercado de capitales local y el de EUA, éste último con mayor rapidez, responden a la incorporación de nueva información lo que a su vez, impacta en la dinámica de la economía doméstica afectando, de alguna forma, los fundamentales macroeconómicos y con su correspondiente efecto sobre las expectativas de los agentes económicos, capturadas hasta cierto punto en el EMBI. Tillmann (2016) documenta la influencia significativa del mercado de EUA, particularmente las decisiones de política monetaria, sobre los EMBI de mercados emergentes, entre ellos México.

Aunque ha crecido el interés por entender cómo se interrelacionan los mercados financieros, no abundan estudios que analicen la influencia del mercado de capitales más importante del mundo, el de Estados Unidos, sobre los mercados de deuda soberana latinoamericana, razón por la cual este trabajo se enfoca en el estudio de los spillovers o efectos entre ese mercado estadounidense y los EMBI Global de algunos de los principales países de América Latina; análisis que se lleva a cabo mediante la metodología propuesta por Diebold y Yilmaz (2009a) y extendida en Diebold y Yilmaz (2012), cuyos elementos más relevantes se describen en la sección siguiente.

\section{Aspectos metodológicos}

De acuerdo con Diebold y Yilmaz (2009b), el índice de spillover que proponen Diebold y Yilmaz (2009a) es de naturaleza muy general y cuenta con gran flexibilidad, basándose directamente en la descomposición de varianza de un modelo de vectores auto regresivos (VAR) con $\mathrm{N}$ variables, el cual es ampliamente conocido y comúnmente utilizado en el análisis de series de tiempo multivariadas. Argumentan dichos autores que el enfoque con que se construye su índice es totalmente diferente de otros, como por ejemplo el propuesto por Edwards y Susmel (2001) el cual produce valores 0 y 1, como indicador de la ubicación del proceso en el estado alto o el estado bajo, y que también están limitados por el 
número de países que pueden incluir. En resumen, el modelo de Diebold y Yilmaz (2009a) representa una ventaja respecto de otras opciones porque su cálculo es relativamente sencillo, dado el herramental disponible para el análisis de series de tiempo multivariadas, y también es más flexible ya que al usarlo se puede considerar en el análisis a un mayor número de países.

Según Diebold y Yilmaz (2012), a pesar de su ventaja sobre otras alternativas, la metodología propuesta por Diebold y Yilmaz (2009a) tiene algunas limitaciones importantes, destacando que la descomposición de varianza puede ser dependiente del orden de las variables analizadas debido a que la identificación del VAR se basa en el enfoque de Cholesky. Otras limitaciones son que la metodología de Diebold y Yilmaz (2009a) únicamente se ocupa de spillovers totales que se dan sólo a través de activos idénticos de diferentes países. Para superarlas Diebold y Yilmaz (2012) usan un enfoque del VAR generalizado en el cual las descomposiciones de la varianza de los errores de los pronósticos son invariantes al orden de las variables, incluyendo además en forma explícita los spillovers direccionales. El punto de partida es un $\operatorname{VAR}(\mathrm{p})$ estacionario en covarianza con $\mathrm{N}$ variables: $x_{t}=\sum_{i=1}^{p} \Phi_{i} x_{t-i}+\varepsilon_{t}, \varepsilon_{t} i i d(0, \Sigma)$, cuya representación como proceso de medias móviles es

$$
x_{t}=\sum_{i=0}^{\infty} A_{i} \varepsilon_{t-i}
$$

en el cual la matriz $A_{i}$, de orden $N \times N$, obedece la recursión $A_{i}=\Phi_{1} A_{i-1}+\Phi_{2} A_{i-2}+$ $\cdots+\Phi_{p} A_{i-p}, A_{0}$ es la matriz identidad también de orden $N \times N$ y $A_{i}=0$ para $i<0$.

Como se ha indicado, la factorización de Cholesky ampliamente utilizada para obtener matrices ortogonales para las innovaciones o choques en el VAR tiene como desventaja la dependencia en el orden de las variables. Para evitar ese problema, Diebold y Yilmaz (2012) recurren a la generalización propuesta por Koop et al. (1996) y Pesaran y Shin (1998) que toma en cuenta adecuadamente la correlación entre los choques utilizando la distribución observada de los errores. Dado que los choques a cada variable no se ortogonalizan, la suma de las contribuciones a la varianza del error de pronóstico no es necesariamente igual a uno.

Las descomposiciones de la varianza del error de pronóstico para $\mathrm{H}$ pasos (periodos) hacia adelante, con $H=1,2, \ldots$, están dadas por

$$
\theta_{i j}^{g}(H)=\frac{\sigma_{i i}^{-1} \sum_{h=0}^{H-1}\left(e_{i}^{\prime} A_{h} \Sigma e_{j}\right)^{2}}{\sum_{h=0}^{H-1}\left(e_{i}^{\prime} A_{h} \Sigma A_{h}^{\prime} e_{j}\right)}
$$

$\Sigma$ es la matriz de varianza del vector de errores del VAR, $\varepsilon_{t}, \sigma_{i i}$ es la desviación estándar del término de error de la i-ésima ecuación y ei es el vector seleccionado con valor unitario en el i-ésimo elemento y cero en los demás. Como se ha señalado, $\sum_{j=1}^{N} \theta_{i j}^{g}(H) \neq 1$, por ello Diebold y Yilmaz (2012) normalizan cada elemento de la matriz de descomposición de varianza sumando por fila

$$
\widetilde{\theta}_{i j}^{g}(H)=\frac{\theta_{i j}^{g}(H)}{\sum_{j=1}^{N} \theta_{i j}^{g}(H)}
$$

resultando por construcción $\sum_{j=1}^{N} \theta_{i j}^{g}(H) \neq 1$ y $\sum_{i, j=1}^{N} \widetilde{\theta}_{i j}^{g}(H)=N$, siendo con base en ello posible construir el índice del spillover total como

$$
S^{g}(H)=\frac{\sum_{i, j=1 ; i \neq j}^{N} \widetilde{\theta}_{i j}^{g}(H)}{\sum_{i, j=1}^{N} \widetilde{\theta}_{i j}^{g}(H)} \bullet 100=\frac{\sum_{i, j=1 ; i \neq j}^{N} \widetilde{\theta}_{i j}^{g}(H)}{N} \bullet 100,
$$

que es análogo al de Diebold y Yilmaz (2009a) que mide la contribución de los spillovers de los choques de todos los activos a la varianza total del error del pronóstico. 
Aprovechando la invarianza respecto del orden de las variables, Diebold y Yilmaz (2012) proponen la medición de los spillovers direccionales, siendo los que recibe el mercado $i$ provenientes de los otros mercados $j$

$$
S_{i \leftarrow}^{g}(H)=\frac{\sum_{j=1 ; j \neq i}^{N} \widetilde{\theta}_{i j}^{g}(H)}{\sum_{j=1}^{N} \widetilde{\theta}_{i j}^{g}(H)} \bullet 100
$$

De manera semejante, los spillovers que transmite el mercado $i$ a los otros mercados $j$ están dados por

$$
S_{i \rightarrow}^{g}(H)=\frac{\sum_{j=1 ; j \neq i}^{N} \widetilde{\theta}_{j i}^{g}(H)}{\sum_{j=1}^{N} \widetilde{\theta}_{j i}^{g}(H)} \bullet 100
$$

Grosso modo, para inicializar la instrumentación de la metodología descrita en esta sección, dado un conjunto de $\mathrm{N}$ series de tiempo con $\mathrm{T}$ observaciones de cada serie, se selecciona un horizonte temporal para estimar el primer VAR, digamos la secuencia $\left(y_{1}, \ldots, y_{t 1}<T\right)$, por ejemplo, en este estudio se utiliza $t_{1}=200$ observaciones diarias de todos los índices sujetos a análisis. A partir de las estimaciones de dicho VAR, se estiman los errores del pronóstico $H$ pasos adelante (los diez días en la aplicación empírica de la sección siguiente que corresponden a dos semanas de operación de los mercados financieros), mediante los cuales se construye el índice del spillover total y sus componentes direccionales con base en la metodología de Diebold y Yilmaz (2012). Después se aplica el mismo procedimiento a los subconjuntos de datos que resultan de un proceso recursivo utilizando una ventana corrediza en la cual se parte ahora de la secuencia $\left(y_{1+H}, \ldots, y_{t 1+H}<T\right)$ hasta agotar la información disponible.

\section{Análisis de los datos}

De las bases de datos de Bloomberg se obtuvo la información diaria de cierre de los índices EMBI Global para Argentina, Brasil, Colombia, México y Perú; las observaciones del S\&P500 se obtuvieron de Yahoo Finance. Las series cubren el periodo entre el 31 de diciembre de 1997 y el 12 de abril de 2017, sumando en total 5,070 observaciones por país; en la figura 1 se puede ver su comportamiento. Destaca en el caso de Argentina de 2001 a 2005 por el elevado nivel de la serie, que lo lleva de una magnitud similar a la de los otros mercados a un alto crecimiento. También es de destacarse que en todos los países latinoamericanos se puede observar alzas en los niveles asociados con el periodo de la crisis financiera en Estados Unidos, reflejada como una severa caída en los valores del índice bursátil de ese país.
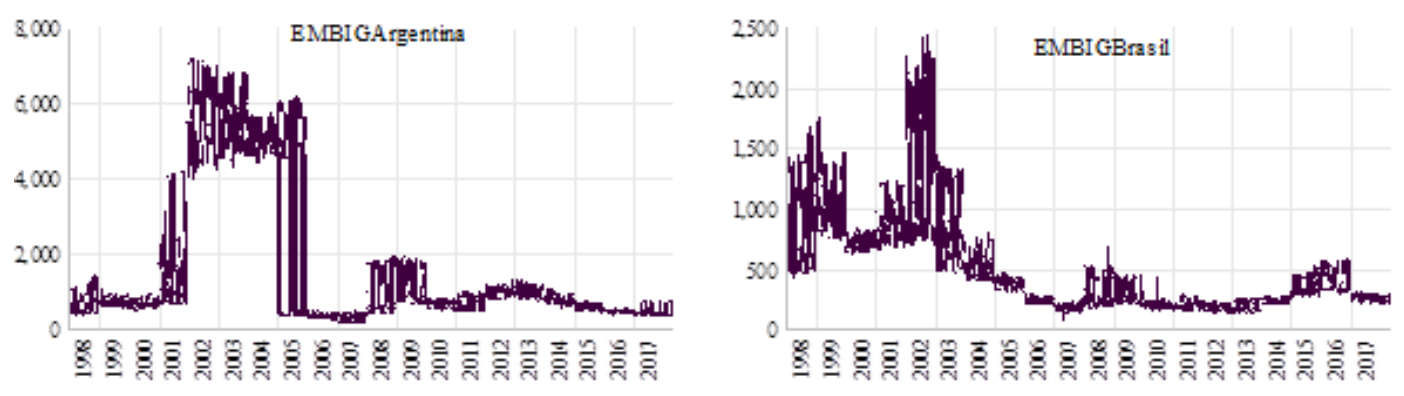

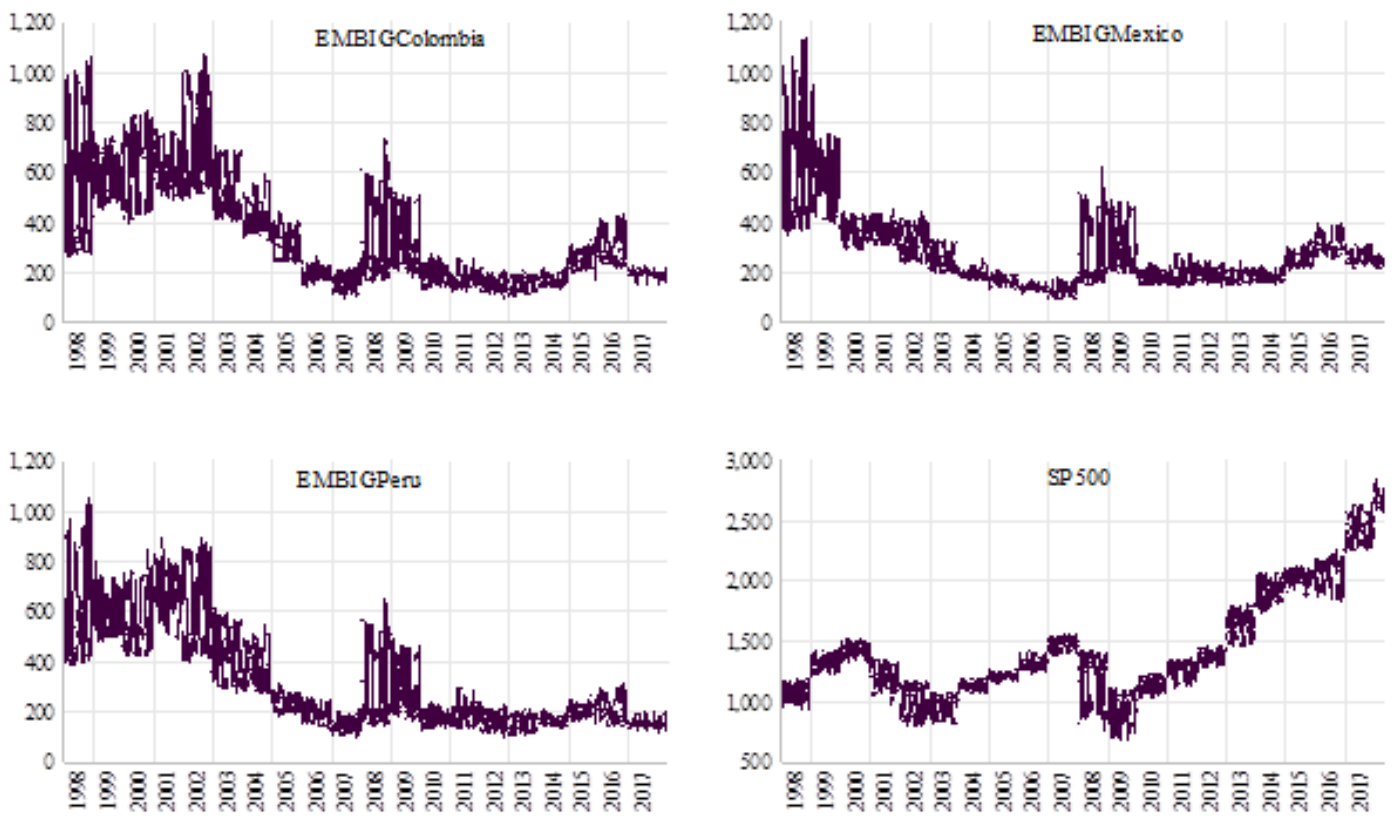

Figura 1. EMBIG y S\&P500

Fuente: Elaboración propia con datos de Bloomberg y Yahoo Finance

Las pruebas ADF de raíces unitarias (cuadro 1) muestran que ninguna de las series de los EMBIG ni el logaritmo del indicador bursátil estadounidense puede considerarse estacionaria, pero sus primeras diferencias sí.

Cuadro 1. Pruebas ADF de raíces unitarias

\begin{tabular}{|c|c|c|c|c|c|c|c|c|c|c|c|c|c|}
\hline \multirow{4}{*}{ Niveles } & & \multicolumn{2}{|c|}{ Argentina } & \multicolumn{2}{|c|}{ Brasil } & \multicolumn{2}{|c|}{ Colombia } & \multicolumn{2}{|l|}{ México } & \multicolumn{2}{|l|}{ Perú } & \multicolumn{2}{|c|}{ S\&P 500} \\
\hline & $\mathrm{C}$ & -1.38 & & -1.61 & & -1.59 & & -1.99 & & -1.61 & & 0.37 & \\
\hline & $\mathrm{CT}$ & -1.80 & & -2.29 & & -3.05 & & -2.00 & & -2.51 & & -0.70 & \\
\hline & $\mathrm{SCT}$ & -1.04 & & -1.26 & & -0.97 & & -1.26 & & -1.43 & & 2.01 & \\
\hline & $\mathrm{C}$ & -19.79 & *** & -14.77 & $* * *$ & -19.86 & $* * *$ & -20.70 & $* * *$ & -17.63 & $* * *$ & -24.10 & $* * *$ \\
\hline Primeras & $\mathrm{CT}$ & -19.80 & $* * *$ & -14.77 & $* * *$ & -19.87 & $* * *$ & -20.71 & $* * *$ & -17.63 & $* * *$ & -24.13 & $* * *$ \\
\hline & SCT & -19.79 & $* * *$ & -14.77 & $* * *$ & -19.86 & $* * *$ & -20.70 & $* * *$ & -17.62 & $* * *$ & -24.00 & $* * *$ \\
\hline
\end{tabular}

Siguiendo la metodología de Diebold y Yilmaz (2012), enunciada en la sección previa, se estimó el índice de spillover entre los rendimientos del S\&P500 y los cambios en los EMBI Global para los países incluidos en el estudio. Mediante la especificación de un modelo vectorial autorregresivo (VAR), se llevó a cabo una estimación de ventana corrediza de 200 días, analizando la varianza del error de pronóstico a diez periodos hacia adelante. En primera instancia, dicha metodología permite construir a partir de ese análisis los efectos de los choques pasados de una variable sobre su propia varianza, así como la contribución (spillover) que hacen los choques en cada variable a la varianza de las otras variables. Adicionalmente, tomando en cuenta ambos efectos, se puede construir el índice de spillover total (véase la figura 2).

Como se observa en la figura 2, después de descender durante el periodo posterior a la crisis del mercado de deuda rusa en 1998 hasta 2001, el índice de spillover vuelve a ascender manteniéndose en un rango de variación entre un poco más de 55 y alrededor de 70. Durante 2006 observó un descenso notorio en ese índice, pero durante 2007 se ubica nuevamente en los niveles previos para continuar durante 2008 un ascenso notorio que lo lleva durante el periodo de 2008 y 2009 a los máximos de todo el periodo. Después de 
la efervescencia asociada con la crisis financiera mundial, se vuelve a ubicar en niveles medios alcanzados en los primeros años del siglo.

En el cuadro 2 se pueden observar la descomposición del índice de spillover en sus componentes direccionales. Se puede ver que un choque en el mercado bursátil estadounidense afecta en $96.4 \%$ al error de pronóstico a 10 semanas de sus rendimientos. Ese mismo choque puede afectar de manera notable al error de pronóstico de los cambios de los EMBIG de todos los países latinos, siendo el más fuerte efecto en el caso de Perú y al que menos afecta a Argentina. Después del mercado bursátil estadounidense, los choques en los cambios de Brasil son otra fuente importante de spillover afectando también de forma notable a los cambios en el EMBIG de Perú, país que es el más afectado por los demás en tanto que el mercado accionario de Estados Unidos es el que se ve menos afectado por los otros. El caso de México es digno de considerarse dado que, después de Perú, es el más afectado por otros, principalmente por Brasil y el S\&P500. Los menos afectados son el mercado accionario de Estados Unidos y el EMBIG de Argentina, en ese orden.

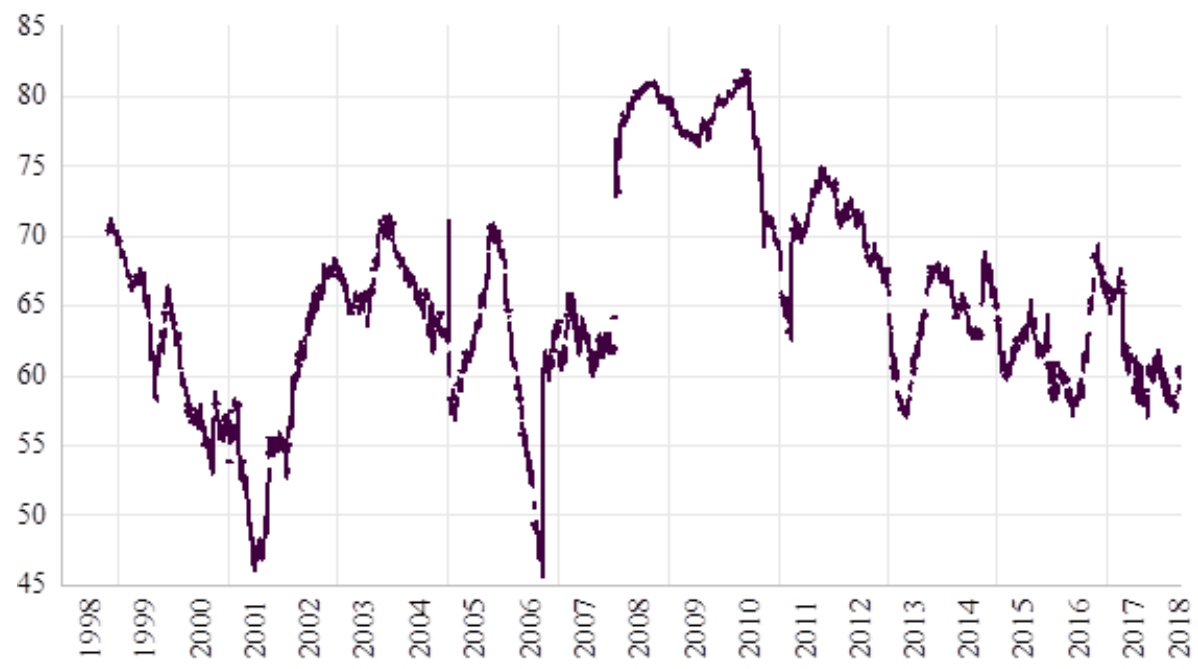

Figura 2. Índice de spillover

Fuente: Elaboración propia con base en estimaciones realizadas en E-Views V10+

Cuadro2. spillover direcional

\begin{tabular}{|c|c|c|c|c|c|c|c|}
\hline & RSP500 & Argentina & Brasil & Colombia & México & Perú & De otros \\
\hline RSP500 & 96.4 & 0.5 & 1.5 & 0.6 & 0.4 & 0.6 & 3.6 \\
\hline Argentina & 8.5 & 89.7 & 0.9 & 0.4 & 0.4 & 0.2 & 10.3 \\
\hline Brasil & 23.6 & 2.2 & 71.3 & 1.6 & 0.4 & 0.8 & 28.7 \\
\hline Colombia & 23.2 & 1.7 & 25.7 & 48 & 0.1 & 1.3 & 52 \\
\hline México & 26.3 & 0.7 & 25 & 17.7 & 29.4 & 0.9 & 70.6 \\
\hline Perú & 29.1 & 1 & 31.2 & 16.8 & 1.1 & 20.9 & 79.1 \\
\hline Contribución a otros & 110.7 & 6.1 & 84.2 & 37.2 & 2.3 & 3.8 & 244.4 \\
\hline
\end{tabular}

Fuente: Elaboración propia con estimaciones realizadas en E-Views V10+

Siendo el mercado accionario estadounidense quien más influencia ejerce sobre los índices de bonos soberanos latinoamericanos incluidos en el análisis, al mismo tiempo que el menos influido por estos mercados, parece conveniente analizar los efectos de derrame (spillover) de su dinámica sobre la dinámica de esos índices de bonos. En la figura 3 se puede observar de inmediato que el comportamiento de los rendimientos de los bonos latinoamericanos responde a los choques que se presentan en el mercado accionario de Estados Unidos prácticamente de manera similar, aunque con magnitudes distintas. En particular destacan como periodos con niveles altos de derrame en todos los índices de bonos latinoamericanos los alcanzados en octubre-noviembre de 2002, diciembre de 2003 a mayo de 2004, febrero de 2009, principios de mayo de 2010, últimos días de diciembre 
de 2012 y fines de febrero de 2017. Cabe destacar que en la mayor parte de esos lapsos de tiempo el índice de los bonos soberanos de Brasil fue el que acusó efectos más elevados.

Otros episodios notorios de derrame, aunque podríamos considerarlos como de impacto mediano, ocurrieron a finales de 1999, los primeros días de enero de 2006, primeros días de abril de 2007, así como a finales de enero e inicios de septiembre de 2014. Es interesante ver como para Argentina se estima un nivel más alto en comparación con los otros índices latinos durante el periodo comprendido de mediados de 2014 a mediados de mayo de 2015 , en tanto que para una buena parte del periodo total analizado se encuentra en niveles comparativos muy bajos. En el caso del índice mexicano destaca que el nivel de derrame estimado para finales de enero de 2009 supera a los índices sudamericanos, aunque no por mucho, en tanto que inicia un movimiento al alza a partir de mediados de agosto de 2017, alcanzando un nivel que supera notoriamente a todos los otros índices de bonos y lo mantiene por arriba incluso hasta el final del periodo analizado.
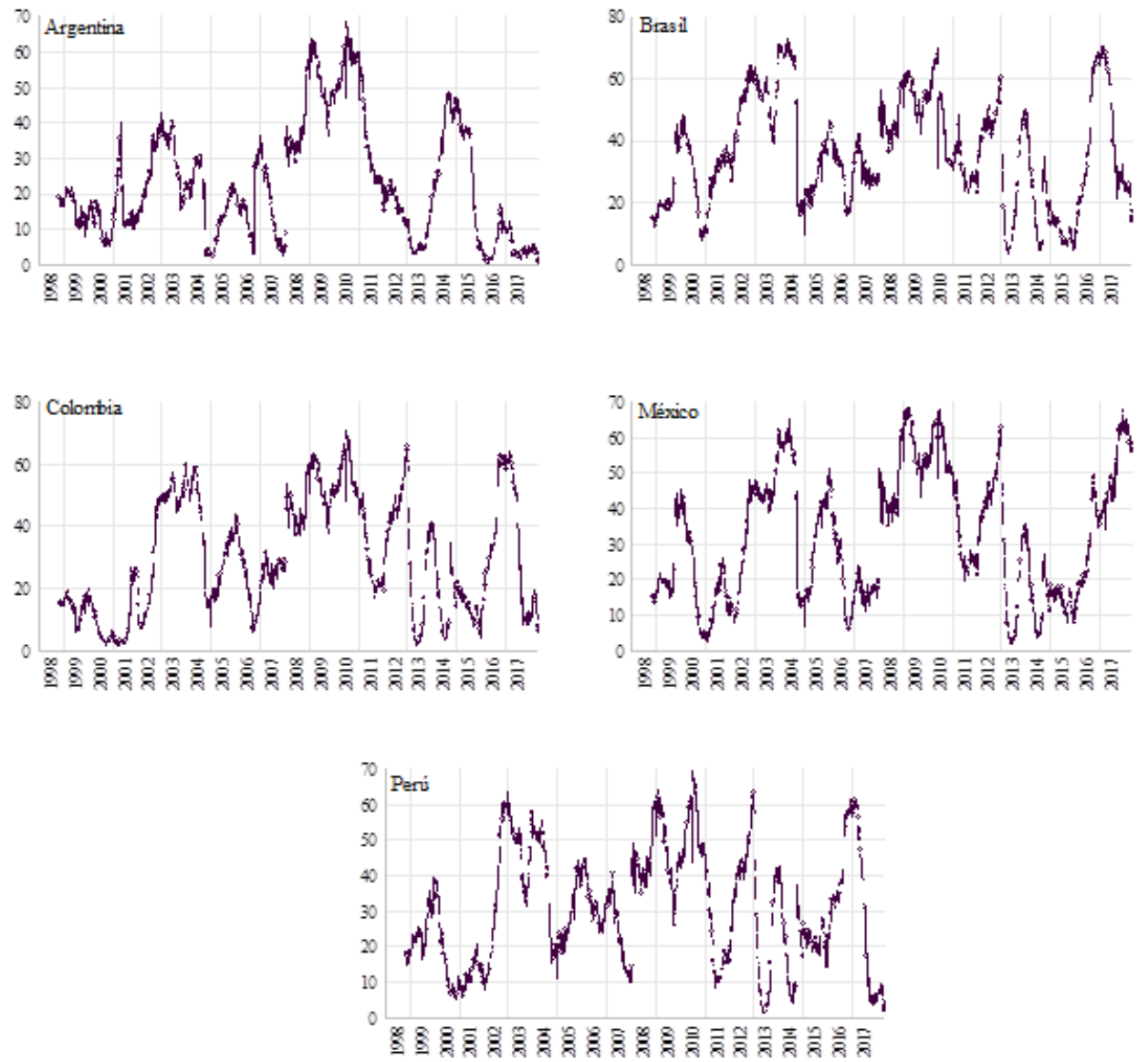

Figura 3. Efectos spillover derivados del SP\&500

Fuente: Elaboración propia con estimaciones realizadas en E-Views V10+ 


\section{Conclusiones}

En este trabajo se analizan los spillovers entre los rendimientos accionarios de Estados Unidos y los cambios en los índices EMBI Global de Argentina, Brasil, Colombia, México y Perú. El índice construido para tales efectos muestra alzas importantes particularmente durante el periodo relacionado con la ocurrencia la crisis subprime iniciada en los mercados financieros estadounidenses.

De acuerdo con los resultados de este análisis, es importante destacar que los choques en el mercado accionario de Estados Unidos, así como el comportamiento del EMBI Global de Brasil son las fuentes más importantes de spillovers. Sin embargo, aunque pequeño en magnitud, también existe un efecto de spillovers de los índices EMBI Global de los países latinoamericanos considerados en este estudio hacia ese mercado accionario, siendo Brasil el que más contribuye. Es bastante probable que esta situación pueda explicarse en gran medida por los efectos del comportamiento de los mercados de deuda latinoamericana y las decisiones de portafolio de fondos de inversión que cotizan en el mercado estadounidense.

Para el caso mexicano es importante considerar que el índice de sus bonos se encuentra, después de Perú, sujeto a spillovers derivados de choques en los otros mercados, principalmente del mercado estadounidense, que dan cuenta hasta un $70.6 \%$ del total de la varianza de su error de pronóstico. Es decir, es un importador neto de los choques externos.

Si bien la evidencia que se ha mostrado en este artículo muestra las principales fuentes de efectos de derrame (spillovers) sobre el comportamiento de los índices de bonos EMBI de algunos de los países latinoamericanos, queda como tarea pendiente para futuras investigaciones ahondar el análisis de forma tal que se puedan determinar otras fuentes de ese tipo de efectos. 
Revista Mexicana de Economía y Finanzas Nueva Época, Vol. 14 Primer Número Especial Aniversario, pp. 527-540

\section{Referencias}

Agliardi, E., Agliardi, R., Pinar, M., Stengos, T., y Topaloglou, N. (2012). A new country risk index for emerging markets: A stochastic dominance approach. Journal of empirical finance, 19(5), 741-761.

Almansour, A., Aslam, A., Bluedorn, J., y Duttagupta, R. (2015). How vulnerable are emerging markets to external shocks? Journal of Policy Modeling, 37(3), 460-483.

Bielschowsky, R. (2009). Sesenta años de la CEPAL: estructuralismo y neoestructuralismo. Revista Cepal, 97, 179-194.

Calvo, S., y Reinhardt, C. (1996). Capital flows to Latin America: Is There Evidence of Contagion Effects? Policy Research Working Paper 1619.The World Bank, International Monetary Fund. http://dx.doi.org/10.1596/1813-9450-1619.

Caporale, G., M., Carcel, H y Gil-Alana, L. A. (2015). The EMBI in Latin America: Fractional Integration, Non-linearities and Breaks. Brunel University London. Department of Economics and Finance, Working Paper No. 15-30.

Cermeño, B., R., y Solís, M., P. (2012). Impacto de sorpresas macroeconómicas de México y Estados Unidos sobre el mercado accionario mexicano. Economía mexicana. Nueva época, 21(1), 35-67

Cimoli, M., y Correa, N. (2005). La apertura comercial y la brecha tecnológica en América Latina: una trampa de bajo crecimiento. Más allá de las reformas: dinámica estructural y vulnerabilidad macroeconómica-Bogotá: Alfaomega/CEPAL, 51-78.

Diebold, F. X. y Yilmaz, K. (2009a). Measuring financial asset return and volatility spillovers, with application to global equity markets. Economic Journal, 119 (534), 158-171.

Diebold, F. X. y Yilmaz, K. (2009b). Efectos derrame en los mercados de valores del continente americano. Economía Chilena, 12 (2), 55-65.

Diebold, F. X. y Yilmaz, K. (2012). Better to give than to receive: predictive directional measurement of volatility spillovers. International Journal of Forecasting, 28 (1), 57-66.

Dimic, N., Kiviaho, J., Piljak, V., y Äijö, J. (2016). Impact of financial market uncertainty and macroeconomic factors on stock-bond correlation in emerging markets. Research in International Business and Finance, 36, 41-51.

Edwards, S. y R. Susmel (2001). Volatility dependence and contagion in emerging equity markets. Journal of Development Economics 66 (2), 505-32.

Forbes, K. J., y Rigobon, R. (2002). No Contagion, Only Interdependence: Measuring Stock Market Comovements? Journal of Finance, 57(5): 2223- 2261. http://dx.doi.org/10.1111/0022-1082.00494

Frenkel, R. (2003). Globalización y crisis financieras en América Latina. Revista de la CEPAL, No. 80, 41-54.

Godfrey, S. y Espinosa, R. 1996. A practical approach to calculating costs of equity for investments in emerging markets, Journal of Applied Corporate Finance, 9(3) 80-89.

Hassan, G. M., y Wu, E. (2015). Sovereign credit ratings, growth volatility and the global financial crisis. Applied Economics, 47(54), 5825-5840.

Hernández, L. F., y Valdés, R. O. (2001). What drives contagion: trade, neighborhood, or financial links? International Review of Financial Analysis, 10(3), 203-218.

Horn, M. P., Hoang, D., Emmel, H., Lahmann, A. D., Gatzer, S., y Schmidt, M. (2017). Country Risk-Cost of Equity Measurement: Methodologies and Implications. Corporate Finance: Finanzierung, Kapitalmarkt, Bewertung, Mergers \& Acquisitions. CF1247951. No. 09-10, pp. 292 - 301.

Iglesias, E. (1992). Reflections on Economic Development; Toward a New Latin American Consensus. Washington, D .C: Inter-American Development Bank.

Koop, G., Pesaran, M.H., y Potter, S.M. (1996), Impulse response analysis in non-linear multivariate models, Journal of Econometrics, 74 (1), 119-147.

Kristjanpoller, R., W., y Salazar A. R. (2016). Inversión extranjera directa y desigualdad en el ingreso en Latinoamérica: evidencia de la cointegración de datos de panel. Cuadernos de Economía, 35(68) 433-455.

Kruger, A. (1980). Trade Policy as an input to Development. American Economic Review, 188-292.

Lessard, D. 1996. Incorporating country risk in the evaluation of offshore projects. Journal of Applied Corporate Finance, 9(3): 5263.

López, H., F., Venegas, M, F., y Gurrola, R, C. (2013). EMBI+ Mexico y su relación dinámica con otros factores de riesgo sistemático: 1997-2011. Estudios Económicos, 28 (2) 193-216.

Mántey, G., y Rosas, E. (2014). EMBI spreads: sentimiento del mercado y fundamentos económicos. Investigación económica, 73(290), 25-50.

Marcet, F. (2017). Analyst coverage network and stock return comovement in emerging markets. Emerging Markets Review, 32, 1-27.

Mellado, C., y Escobari, D. (2015). Virtual integration of financial markets: a dynamic correlation analysis of the creation of the Latin American Integrated Market. Applied Economics, 47(19), 1956-1971.

Mendoza, V., A., López, H., F. y Watkins, F., K. (2011), Reflexiones sobre las crisis financieras, Centro de Investigación e Inteligencia Económica CIIEUPAEP y División de Investigación de la Facultad de Contaduría y Administración, UNAM. Coordinadores. 
Mollah, S., y Hartman, T. (2012). Stock Market Contagion, Interdependence and Shifts in Relationship due to Financial Crisis-A Survey. International Review of Business Research Papers, 8(1), 166-195.

Oliva, R., y Suarez, F. F. (2007). Economic reforms and the competitive environment of firms. Industrial and Corporate Change, 16(1), 131-154.

Oliveira, L., Curto, J. D., y Nunes, J. P. (2012). The determinants of sovereign credit spread changes in the Euro-zone. Journal of International Financial Markets, Institutions and Money, 22(2), 278-304.

Onaran, O. (2009). Wage share, globalization and crisis: the case of the manufacturing industry in Korea, Mexico and Turkey. International Review of Applied Economics, 23(2), 113-134.

Ortiz, E., López, H. F., Santillán, S. R. J., y Fonseca, R. A. (2016). The integration of Latin American bond markets: a copula analysis approach (1999-2015). International Journal of Bonds and Derivatives, 2(3), 267-283.

Özmen, E., y Yaşar, Ö. D. (2016). Emerging market sovereign bond spreads, credit ratings and global financial crisis. Economic Modelling, 59, 93-101.

Pesaran, M.H. y Shin, Y. (1998). Generalized impulse response analysis in linear multivariate models. Economics Letters, 58 (1), 17-29.

Piljak, V. (2013). Bond markets co-movement dynamics and macroeconomic factors: Evidence from emerging and frontier markets. Emerging Markets Review, 17, 29-43.

Rigobon, R (2002). International Financial Contagion: Theory and Evidence in Evolution, CFA. The Research Foundation Publications.

Romero, A. Y. P., Ramírez, A. F. H. y Guzmán, A. D. S. (2013). Mercado Integrado Latinoamericano (MILA): análisis de correlación y diversificación de los portafolios de los tres países miembros en el período 2007-2012. Cuadernos de Contabilidad, 34(14), 53-74.

Sandoval, E., y Soto, M. (2016). Mercado integrado latinoamericano: un análisis de cointegración. Revista Internacional Administración \& Finanzas, 9(2), 1-17.

Santillán, S., R., J. (2015). La Gran Recesión (2007-2012): Lecciones y Oportunidades para México. EGADE Business School-Instituto Mexicano de Ejecutivos de Finanzas, PWC. Roberto J. SantillánSalgado, Coordinador.

Santillán, S., R., J., Gurrola, R., C., Venegas, M., F., y Jiménez, P., A., L. (2018). La dependencia del Índice de Precios y Cotizaciones de la Bolsa Mexicana de Valores (IPC) con respecto a los principales índices bursátiles latinoamericanos. Contaduría y Administración, Disponible en: http://www.cya.unam.mx/index.php/cya/article/view/1256/1247.

Te Velde, D. W. (2003). Foreing direct investment and income inequality in Latin America: Experiences and policy implications. Instituto de Investigaciones Socioeconómicas, Universidad Católica Boliviana.

Tillmann, P. (2016). Unconventional monetary policy and the spillovers to emerging markets. Journal of International Money and Finance, 66, 136-156.

Uribe, J. (2011). Contagio financiero: una metodología para su evaluación mediante coeficientes de dependencia asintótica. Lecturas de Economía, (75), 29-57. 